\title{
Influence of Distribution Size of Micro Energy Director Array on Ultrasonic Precise Sealing of Polymer MEMS Device
}

\author{
Miaomiao Zhang \\ School of Mechatronics Engineering, Zibo vocational college, Zibo, Shandong, China
}

\begin{abstract}
In order to improve the quality and precision of ultrasonic precision sealing of polymer MEMS device, micro energy director array was proposed on the sealing surface of polymer MEMS device, the influence of the distribution size of micro energy director array to ultrasonic precise sealing was studied. Based on the experiment of PMMA micro-pipe sealing, five kinds of micro energy directors with different distribution sizes were made on the sealing surface by hot embossing technique. The method of ultrasonic precise sealing based on efficiency's feedback was used and the influence of micro energy director array's distribution size on the quality of ultrasonic sealing was summarized through the observation on the rule of wetting behavior of micro energy director array.
\end{abstract}

Keywords: Ultrasonic precise sealing; MEMS; energy director; wetting

\section{Introduction}

Polymers are used to manufacture such MEMS devices as microfluidic chips, micro fuel cells, micro valves, micro pumps, and micro sensors thanks to its advantages in low price, low production cost, and easy mass production ${ }^{[1]}$ As increasingly more polymer materials are used in the field of MEMS, precise sealing of polymer MEMS devices has become the key to MEMS manufacturing technology ${ }^{[2]}$.

At present, polymer sealing methods in the MEMS field mainly include thermal bonding ${ }^{[3]}$, glue/solvent bonding ${ }^{[4]}$, laser/microwave welding ${ }^{[5][6]}$, surface modification bonding ${ }^{[7]}$, etc. However, most of these methods face certain problems in sealing efficiency and quality. Ultrasonic sealing technology has been widely used in the MEMS field with its high efficiency, free introduction of other substances, and local heat generation. In 2006, Truckenmüller applied ultrasonic sealing technology for the first time to assemble microfluidic chips, micro pumps and micro valves, which verified the feasibility of ultrasonic sealing technology to micro device packaging ${ }^{[8]}$. In 2009 , Kim achieved sealed connection of cellulose acetate micro-devices with outer diameter and inner diameter of $1.8 \mathrm{~mm}$ and $0.85 \mathrm{~mm}$ respectively ${ }^{[9]}$. In 2018, Lu WANG et al. verified the influence of welding conditions on the welding strength of PC/ABS and PMMA, and proposed the optimal welding parameters ${ }^{[10]}$. The above research has extended ultrasonic welding to the field of polymer MEMS device packaging and verified the feasibility of this technology. However, sensitivity of sealing quality to the input energy lacks in-depth study after the device miniaturization. In macroscopic plastic ultrasonic welding, to increase heat generation rate of the weldment interface for higher efficiency, energy director ribs are usually made on the joint surface ${ }^{[11]}$. For energy director in ultrasonic welding, Rani et al. studied different joint forms, finding that surface with a rough structure has greater sealing strength than larger-sized energy director. Meanwhile, the absorbed energy is smaller, and the micro-device is less deformed ${ }^{[12]}$; Qiu et al. believed that for surface structure with a certain height and roughness, there is a suitable pressure to enable the optimal bonding effect ${ }^{[13]}$; Buckley et al. simplified the rough surface to an ideal rectangular model, simulated the wetting process based on approximation extrusion fluid theory. The research results indicate that polymer diffusion and wetting process is quite sensitive to surface morphology of the sealing surface, and surface with low roughness is easy to cause uneven diffusion on the sealing surface, thus increasing the occurrence probability of defects ${ }^{[14-15]}$.

For MEMS, due to the small dimension of micro devices, traditional energy director rib structure is inapplicable. In the current ultrasonic sealing technology for MEMS devices, energy director is basically not used, but flat joint is

ISSN: 0010-8189

(C) CONVERTER 2020

www.converter-magazine.info 
used for sealing ${ }^{[10]}$. In the same batch of specimens, due to different surface morphology and roughness of the sealing surface, there is certain influence on the contact area, contact mode and contact force of the sealing surface. Hence, the sealing interface has certain differences in heat production rate and heat production area in the ultrasonic sealing process, which incurs certain randomness in the ultrasonic sealing process and in turn affects the sealing precision ${ }^{[16]}$.

Considering that for micro devices, difference in the topography and roughness of the sealing surface will cause a certain difference in heat production rate and heat production area of the sealing interface during the ultrasonic sealing process, which causes certain randomness in ultrasonic sealing process and in turn affects sealing precision, Yibo SUN of Dalian University of Technology, et al. proposed to design a quadrangular pyramid micro energy director array on the sealing surface to replace the energy director ribs of the macro welding surface, verified that micro energy director array can effectively control the spread of molten polymer, increase the utilization efficiency of ultrasonic energy, derive a uniform sealing surface and achieve high-quality precise sealing. It is proposed that small-sized micro energy director array facilitates the spread of molten polymer on the sealing surface, so that precise sealing of micro devices is possible under small ultrasonic energy ${ }^{[17]}$.

To investigate the influence of distribution size of micro energy director on the welding quality, this paper tested specimens V1-V5 of micro energy director array with the same structure size and different distribution size. It examines the influence law of distribution size of micro energy director array on the ultrasonic sealing parameters, wetting behavior and sealing quality.

\section{Design and Manufacture of Micro Energy Director Array}

In macroscopic polymer ultrasonic welding technology, energy director ribs with a triangular cross-section has a smaller contact area compared to other shapes, which can greatly increase the acoustic energy density of the ultrasound and make the joint reach a higher temperature. In view of this feature, this paper designs a rectangular pyramid micro energy director. The micro energy director is fabricated on the substrate sealing surface by using silicon molds and hot embossing. Fig. 1 shows a substrate with micro energy director array fabricated on the surface and a schematic cross-sectional view of the two micro energy directors. $d$ represents the spacing between the micro energy director array, which indicates the distribution size in the experiment.

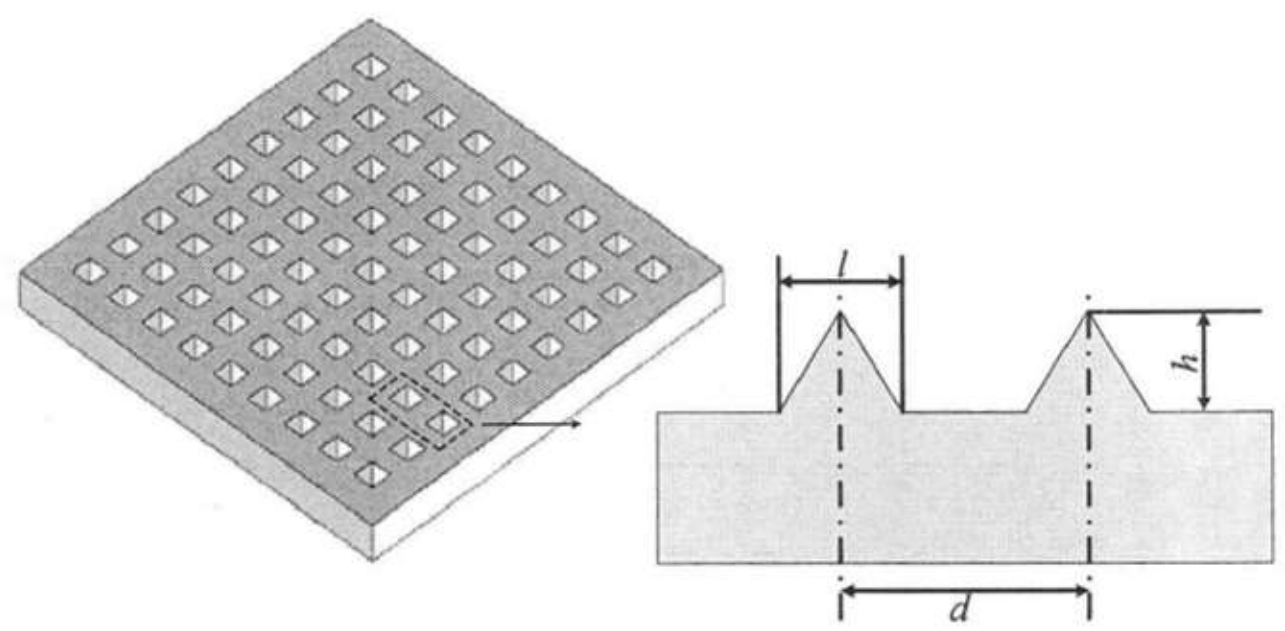

Fig. 1 Schematic diagram of micro energy directors array and section plane

With PMMA (polymethyl methacrylate) micropipe as the experimental device, ultrasonic sealing technology is used to melt and seal it with the substrate. The structure is shown in Fig. 1. The micropipe has an outer diameter of $3 \mathrm{~mm}$ and an inner diameter of $1 \mathrm{~mm}$, and the micro energy director array is fabricated on the substrate surface.

ISSN: 0010-8189

(C) CONVERTER 2020 
PMMA material (Asahi Kasei, Shanghai) was used in the experiment, whose glass transition temperature was about $105{ }^{\circ} \mathrm{C}$, and melting temperature was about $180{ }^{\circ} \mathrm{C}$.

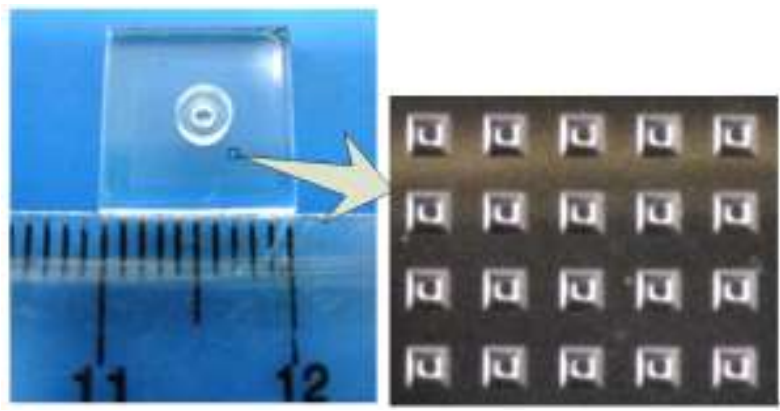

Fig. 2 Substrate with PMMA micro connector and local amplificatory structure

The micro energy director was made by hot embossing, and the selected equipment was RYJ-II hot press for micro-system technology ${ }^{[18]}$. The hot embossing parameters were: hot embossing temperature: $135{ }^{\circ} \mathrm{C}$, pressure: 2.5 $\mathrm{MPa}$, heat preservation and pressure preservation time: $300 \mathrm{~s}$. The mold used for hot embossing was a silicon mold, and the microstructure was made by anisotropic wet etching process ${ }^{[19]}$. The selected silicon wafer was $<100>$ crystal orientation n-type doped silicon (Max, Luoyang), the mold structure size was determined by the window size of the mask plate, the etching solution was $\mathrm{KOH}$ : IPA: $\mathrm{H} 2 \mathrm{O}=40 \mathrm{~g}: 30 \mathrm{ml}: 100 \mathrm{ml}$ (volume ratio), and the etching temperature was $73{ }^{\circ} \mathrm{C}$. In view of the characteristic that the inclination angle of silicon anisotropic corrosion was $54.7^{\circ}$, the microstructure mold of quadrangular pyramid was derived from self-stopped etching, as shown in Fig. 3. The micro energy director by hot embossing is shown in the enlarged part of Fig. 2.

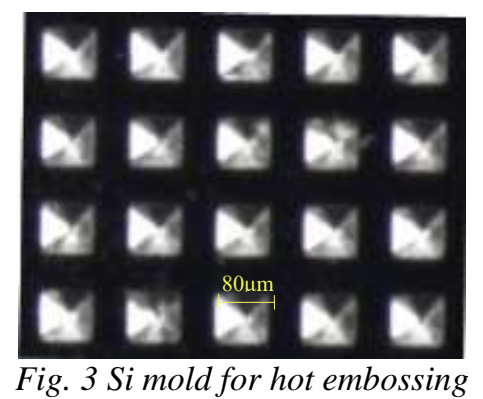

Fig. 1 shows a schematic side view of the microstructure of the PMMA substrate by hot embossing. To study the influence of distribution size of micro energy director array on sealing, this paper designs five groups of micro energy directors in different sizes. The width $l$ of micro energy director in each group is $80 \mu m$, and the spacing $d$ is $80 \mu \mathrm{m}, 160 \mu \mathrm{m}, 240 \mu \mathrm{m}, 320 \mu \mathrm{m}, 400 \mu \mathrm{m}$, respectively. The specimens are marked as I, II, III, IV, V.

\section{Ultrasonic Sealing Experimental Device}

The experimental device of ultrasonic precise sealing is shown in Fig. $4.60 \mathrm{kHz}$ ultrasonic transducer is used to provide high-frequency, low-amplitude ultrasonic vibration. Higher frequency enables sufficiently high heat production rate for the sealing at relatively small amplitude. By reducing the amplitude, it is possible to reduce the mechanical vibration load of micro devices and lower damage to micro device. A high-subdivision stepping motor and a linear guide are used to drive the longitudinal movement of the ultrasonic tool head. A micro-displacement sensor is used to detect a small distance near the contact position of the tool head and the part, which can increase movement precision of the control tool head and apply a stable pressure. The platform bearing the parts is loaded with a pressure sensor for real-time detection of the pressure exerted on the parts by the linear guide drive tool head. 


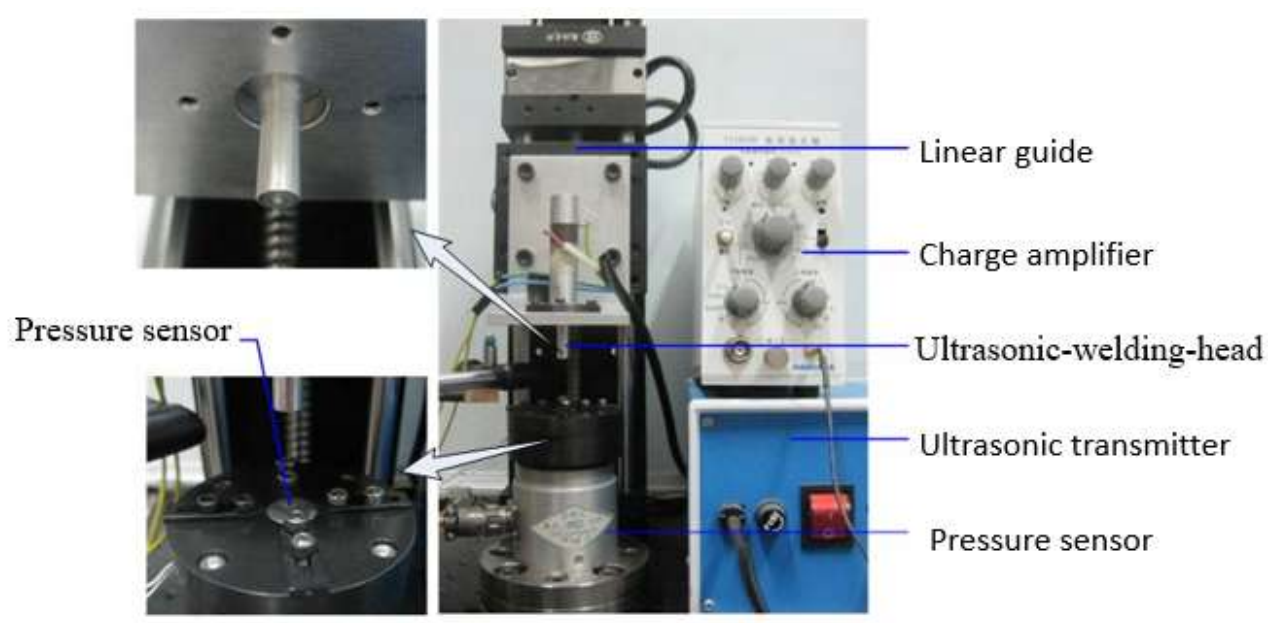

Fig. 4 Ultrasonic precise bonding mechanism

To detect and control the ultrasonic sealing process, the experiment adopts ultrasonic precise sealing method based on the feedback of sound wave transmission efficiency. The mechanism is to detect ultrasonic vibration information generated by the ultrasonic tool head and transmitted to the bottom surface of the substrate through the two layers of polymer materials to be sealed, thus providing feedback and controlling the on-off of ultrasonic energy. During the ultrasonic sealing process, the polymer device has higher interface temperature, which locally changes from glass state to viscoelastic state. As the viscoelastic body generates more intense heat, it turns into a viscous fluid state. As the state changes, the polymer material has greatly reduced elastic modulus ${ }^{[18]}$, and its sound wave transmission efficiency will be greatly attenuated. Since the vibration provided by the ultrasonic tool head remains constant, attenuation degree in sound wave transmission efficiency of polymer device can be reflected by detecting the vibration information transmitted to the substrate bottom surface. To detect the vibration of the substrate bottom surface, a piezoelectric ceramic sensor is installed on the bearing platform, whose control system structure is shown in Fig. 5. The sensor signal is converted into an analog voltage signal by a charge amplifier, and a data acquisition card (Advantech PCI-1712, China) is used to convert it into a digital signal and input it into an industrial computer and control program, thereby controlling the ultrasonic power supply and movement of the branch rails. The entire control flow is implemented by LabVIEW software.

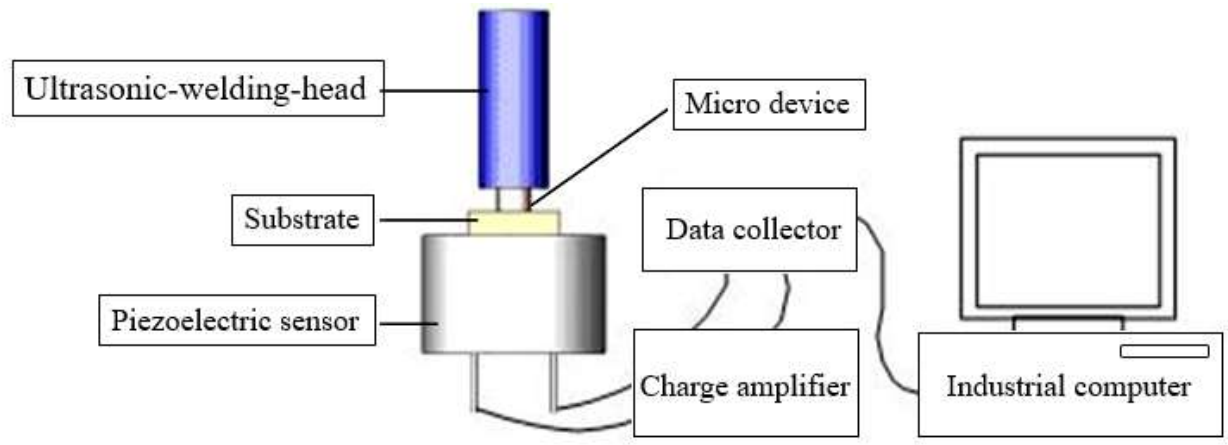

Fig. 5 Principle diagram of ultrasonic bonding based on ultrasound propagation

For the ultrasonic sealing of polymer, at the beginning of ultrasonic application, the polymer is in the glass state, with big initial output voltage $\mathrm{U}_{0}$ transmitted to the piezoelectric sensor as a result of the piezoelectric effect. Under the action of ultrasound, the polymer transforms to a viscoelastic state. At this time, part of the ultrasonic energy transmitted to the substrate bottom through the sealing interface will be stored in the polymer in viscoelastic state. The vibration signal detected by the piezoelectric sensor weakens and the corresponding output voltage value $U$ continues to decrease. Under greater volume of the viscoelastic polymer at the sealing interface, smaller vibration amplitude is detected by the sensor, and the output voltage value is smaller. Therefore, by controlling the attenuation ratio $\rho=\mathrm{U} / \mathrm{U}_{0}$ of the voltage amplitude to achieve on-off time control of the ultrasound,

ISSN: 0010-8189

(C) CONVERTER 2020 
the polymer melting volume can be effectively controlled. The amplitude feedback control mode is adopted for ultrasonic sealing, and on-off of the ultrasound is controlled by mechanical state of the polymer to reflect the variation law of the polymer in the ultrasonic field, and achieve adaptive and precise control of the input energy in the sealing of micro devices, thus offering a feasible method for precise sealing of micro devices.

\section{Experimental Results and Discussion}

Based on the aforementioned devices and control methods, this paper tests substrates of micro energy director array of different distribution sizes listed in I-V. For each specimen, the voltage amplitude attenuation ratio $\rho$ was selected as $80 \%, 70 \%, 60 \%$, and $50 \%$ for the ultrasonic sealing experiment, and the sealing degree of the sealing interface was observed via an optical microscope. The process parameters in the experiment are ultrasonic amplitude A: $6 \mu \mathrm{m}$, ultrasonic frequency f: $60 \mathrm{kHz}$, trigger pressure $\mathrm{F}_{\mathrm{T}}: 15 \mathrm{~N}$, and dwell time $\mathrm{T}_{\mathrm{H}}: 5 \mathrm{~s}$.

Fig. 6 shows the welding effects of micro energy director in different distribution sizes under sealing modes of different voltage amplitude attenuation ratio. The sealing results of each group of specimens are observed under different voltage amplitude attenuation parameters. Take group I experiment as an example. Under the sealing parameter with voltage amplitude attenuation ratio at $80 \%$, the micropipes fall off and the sealing is not achieved. From the sealing area of the fallen pipes, it can be seen that only a small part of the micro energy director has melted, while most of it is not melted, so effective sealing is impossible. Under the sealing parameter with voltage amplitude attenuation ratio at $70 \%$, the micropipes still fall off and the sealing is not achieved. Nevertheless, it can be seen from the sealing area that the micro energy director has significantly increased melting area. Under the sealing parameter with voltage amplitude attenuation ratio at $60 \%$, the micropipe is successfully sealed, but there is still a small part of the micro energy director not melted on the sealing surface; under the sealing parameter with voltage amplitude attenuation ratio at $50 \%$, the micropipe is effectively sealed, with only a small amount of micro energy director not melted on the sealing surface.

The same rule also appeared in the II-V group experiment. That is, for each group, as the attenuation ratio decreased from $80 \%$ to $40 \%$, the molten polymer exhibited an increasing trend. For its reason, it is because in the ultrasonic sealing process, the polymer in contact between the sealing surfaces heats up and melts under high-frequency mutual friction, and the molten polymer absorbs part of the ultrasonic energy transmitted by the welding head, resulting in attenuated transmission of the ultrasonic vibration to the substrate bottom surface. Under greater melt volume, greater ultrasonic energy is absorbed, and there is greater vibration attenuation of the substrate bottom surface.

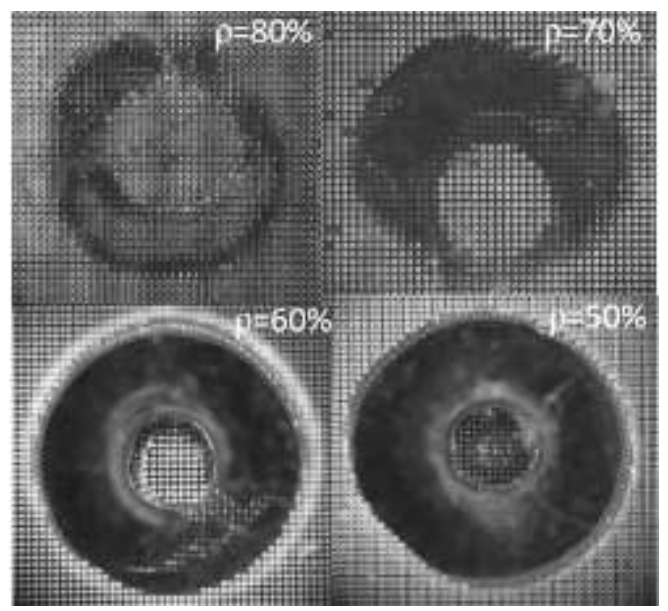

a. specimen I

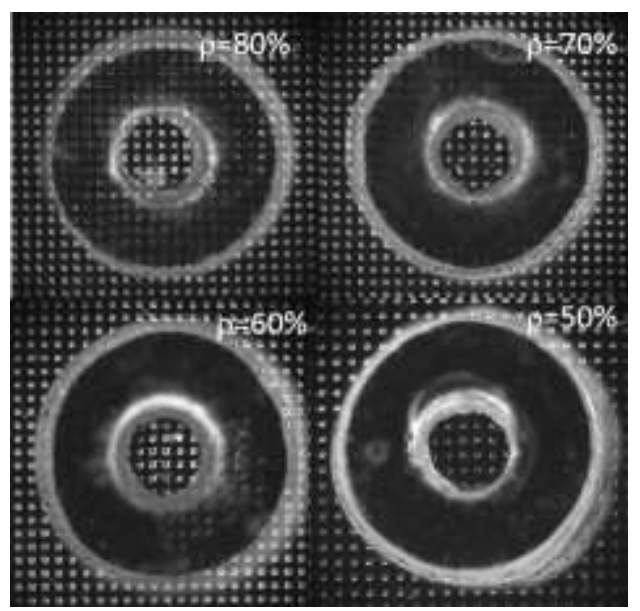

b. Specimen II

ISSN: 0010-8189

(C) CONVERTER 2020 


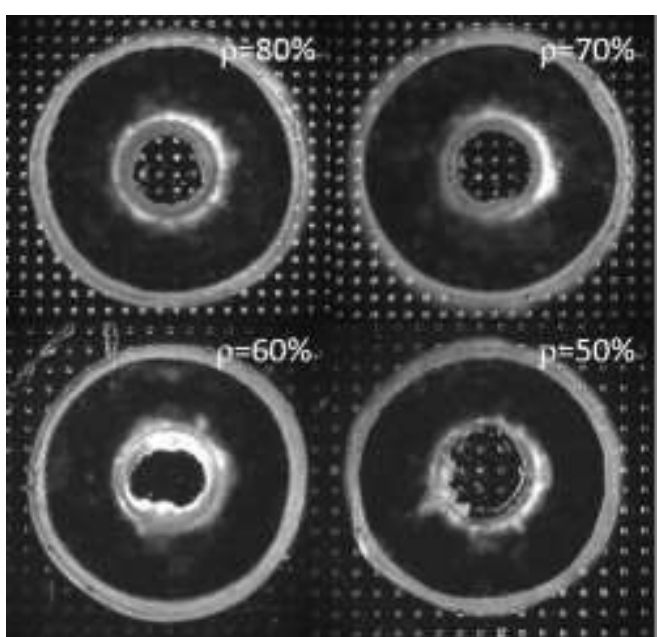

c. specimen III

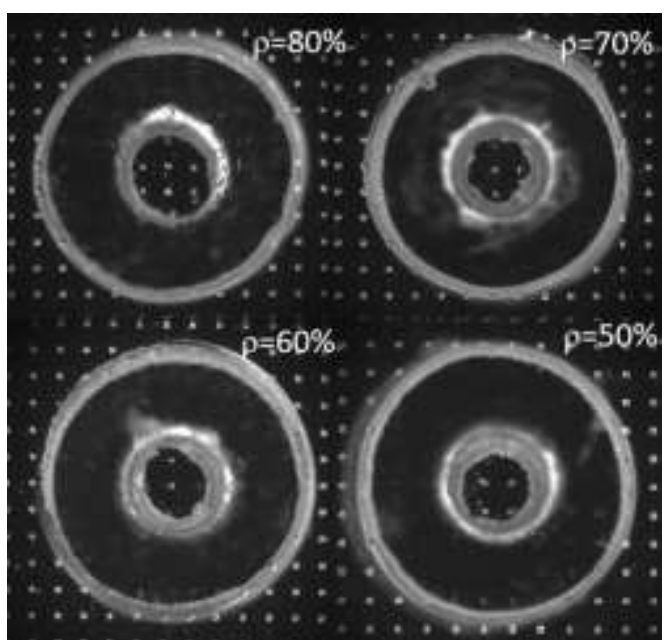

d. Specimen IV

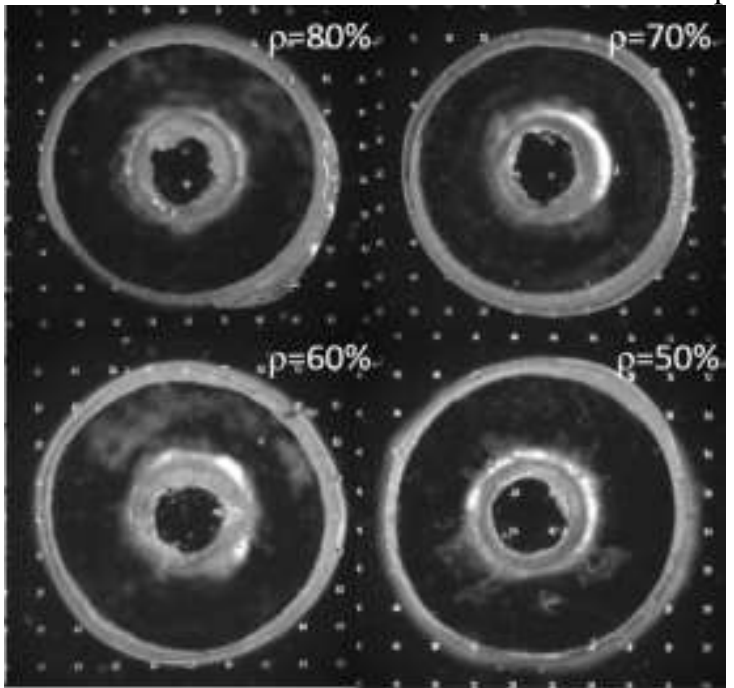

e.V specimen

Fig.6 Fusion interface of specimens I-Vobtained by ultrasonic bonding at various parameters

Regarding the influence of distribution size of the micro energy director to be studied on sealing quality, we analyze the experimental results from another perspective: under the same parameter conditions, analyze welding status of the micro energy director with different distribution sizes. Under $80 \%$ voltage amplitude attenuation ratio, most of the micro energy director of specimen I was not melted, and the micropipes were not be sealed; specimen II with increased distribution size achieved partial welding, but there were still areas where micro energy director was not completely collapsed. With the further increase of the array spacing, specimens III-V basically achieved complete fusion of the interface, and the surface micro energy director was also completely collapsed and evenly spread on the entire interface. Under $70 \%$ voltage amplitude attenuation ratio, the micro energy director of specimen I was not been completely melted, and the micropipes were not sealed. Specimen II-V achieved sealing of different degrees, and specimen III had optimal sealing surface quality. The same rule still existed under $60 \%$ and $50 \%$ voltage amplitude attenuation ratio. Specimens with better sealing surface quality were specimen III and specimen II.

To conclude, we can see that:

a. As spacing of the micro energy director increases, interface welding degree gradually increases.

The micro energy director arrays on the surface of the specimens I-V have consistent structural size, but the difference lies in distribution spacing. Therefore, for polymer MEMS devices, when the micro energy director

ISSN: 0010-8189

(C) CONVERTER 2020

www.converter-magazine.info 
array has consistent structural size, increasing the distribution spacing is equivalent to reducing the number of micro energy directors per unit area. According to the ultrasonic precise coupling method based on the feedback of ultrasonic propagation efficiency, the amount of polymer whose local mechanical state of the interface micro energy director undergoes melting changes is theoretically consistent with the amount of ultrasonic energy absorbed. Therefore, under the same parameter conditions, as the distribution distance increases, fewer micro energy directors between the interfaces will be melted, leading to higher melting degree. Accordingly, interface welding degree gradually increases under the same voltage amplitude attenuation ratio for specimens I-V.

b. There are certain differences in the optimal parameters when micro energy directors with different distribution sizes achieve ideal sealing.

This phenomenon reflects the wetting and spreading law of the melt of the micro energy director. The melt flow in the ultrasonic welding process exhibits pressure compression behavior of the polymer. At the same time, because of the big viscosity of the polymer melt, the wetting of the sealing part surface by the melt mainly depends on extrusion flow of the melt. In the initial stage of ultrasonic sealing, high-frequency friction occurs between the micro energy director and the contact part of the other surface. When the interface temperature exceeds the polymer melting temperature, the molten area will flow under the action of pressure and ultrasonic load. The extruded molten polymer spreads continuously between the upper and lower surfaces, finally forming a welded layer for sealing of the interface. Spreading degree of the molten polymer directly determines the sealing surface quality to a certain extent. When the spacing between the micro energy director is small, that is, the number of micro energy director per unit area is high and the contact area between the polymer conjunction plane is big, to realize complete melting of the micro energy director, it is necessary to increase the ultrasonic energy so that complete spread of the molten polymer between the sealing surfaces is possible. Conversely, when the spacing between the micro energy director is big, a smaller ultrasonic energy is needed to achieve complete spread of the molten polymer between the sealing surfaces.

c. With the decrease of the voltage amplitude attenuation ratio, there are unobvious microbubbles caused by the ultrasonic cavitation effect between the interface of specimens II-IV, while as the voltage amplitude attenuation ratio decreases, bubbles can be found between the interface of specimen $\mathrm{V}$.

There are no large-area microbubbles in the welding interface of specimens II-IV, but with the further increase of the spacing, it is found that more microbubbles are gradually produced as the voltage amplitude attenuation ratio of specimen V decreases. Different from planar ultrasonic connection, manufacture of micro energy director array replaces large-area contact with a small contact area. The melting and spreading of the structure in local area reduces the material extrusion degree and increases the polymer casting during the welding process, which facilitates polymer spreading and gas overflow, thus inhibiting ultrasonic cavitation effect to a certain extent. However, as the distribution size increases, there are fewer micro energy directors per unit area. Under the action of ultrasound, the micro energy director collapses and spreads between the interfaces. Owing to the small amount of spreading material, complete spreading is too early under the same parameters, so ultrasound acts on the polymer material of the entire interface, which makes it difficult to suppress ultrasonic cavitation.

\section{Conclusions}

In this paper, micro energy director array is proposed for ultrasonic precise sealing of polymer MEMS devices. Five groups of rectangular pyramid-shaped micro energy director arrays with different distribution sizes are fabricated by silicon mold hot embossing. A series of ultrasonic sealing experiments were carried out on micro devices with different distribution sizes under different voltage amplitude attenuation ratios. The wetting behavior of the interface polymer during the ultrasonic sealing process was observed and analyzed to study the influence of distribution size of micro energy director on ultrasonic sealing quality. The results further verified that this kind of micro energy director can eliminate the influence of microdevice surface morphology and roughness on sealing quality, effectively control the polymer melt casting, and result in a good sealing surface, which forms mutual

ISSN: 0010-8189

(C) CONVERTER 2020

www.converter-magazine.info 
corroboration with research results of Yi LUO et al. of University of Technology ${ }^{[17]}$. The research results suggest that bigger distribution size of the micro energy director reduces the demand of complete welding of the sealing interface for ultrasonic energy, which also exerts certain inhibitory effect on the micro-bubbles caused by ultrasonic cavitation. However, excessive increase in spacing will cause casting of the micro energy director, so that it is impossible to fully spread over the entire interface, making it difficult to optimize the casting of polymer materials. Yanguo ZHANG ${ }^{[20]}$ found in microfluidic chip sealing experiment that the surface energy director of the microfluidic chip must be designed with a reasonable structure and size to guarantee a good chip sealing. This experiment clarifies the method to obtain a good sealing surface from the perspective of designing reasonable spacing of the micro energy director. Yibo SUN et al. verified the influence of the size of the micro energy director on the polymer ultrasonic sealing quality in experiments. The conclusions of this experiment form a complementary relation with it. It more comprehensively studies the influence of surface morphology and roughness of the microdevice on sealing quality. Therefore, in terms of manufacture of micro energy director arrays, it is necessary to select appropriate distribution sizes, so that by combining appropriate structural size, it is possible to inhibit the ultrasonic cavitation of the interface and increase the interface welding strength and sealing.

\section{References}

[1] J. Jie, T. Wang, C.G. Wang, W.B. Wu, L.Y. Shuai, L.Z. Wang, "Highly precise Ti/Pt/Cr/Au thin-film temperature sensor embedded in a microfluidic device," Rare Metals, vol. 40, no. 01, 2021.

[2] S.J. Lee, "Micro total analysis system ( $\mu$-TAS) in biotechnology," Applied Microbiology and Biotechnology, vol. 64, pp. 289-299, 2004.

[3] U. Masanori, S. Toshikazu, K. Michiaki, et al., Reliability of Cu Nanoparticles/Bi-Sn Solder Hybrid Bonding Under Cyclic Thermal Stresses, vol. 73, no. 2, pp. 600-608, 2021.

[4] R. Sivakumar, N.Y. Lee, "Microfluidic device fabrication mediated by surface chemical bonding," vol. 145, no. 12, pp. 4096-4110, 2020.

[5] M. Chambonneau, Q.F. Li, Fedorov Vladimir Yu., et al., "Ultrafast Laser Welding: Taming Ultrafast Laser Filaments for Optimized Semiconductor-Metal Welding," Laser Photonics Rev, vol. 15, no. 2, 2021.

[6] Nanotechnology - Nanostructures; Studies from Old Dominion University Provide New Data on Nanostructures (Spray-Assisted Microwave Welding of Thermoplastics Using Carbon Nanostructures with Enabled Health Monitoring), pp. 2033, 2019.

[7] M. Bednarik, A. Mizera, M. Manas, et al., "Influence of the $\beta<$ sup $>_{-}</$sup $>$Radiation/Cold Atmospheric-Pressure Plasma Surface Modification on the Adhesive Bonding of Polyolefins," vol. 14, no. $1,2020$.

[8] R. Truckenmüller, R. Ahrens, Y. Cheng, et al., "An Ultrasonic welding based process for building up new class of inert fluidic microsensors and actuators from polymers," Sensors and Actuators, vol. 132, no. 1, pp. 385-392, 2006.

[9] J. Kim, B. Jeong, M. Chiao, "Ultrasonic bonding for MEMS sealing and packaging," IEEE Transactions on Advanced Packaging, vol. 32, no. 2, pp. 461-467, 2009.

[10] S.H. Ng, Z.F. Wang, ROOIJ N F DE, "Microfluidic connectors by ultrasonic welding," Microelectronic Engineering, vol. 86, no. 4-6, pp. 1354-1357, 2009.

[11] LIU CH, "The mechanism of ultrasonic plastic welding and the study of experiments," Dalian: Dalian University of Technology, 2003.

[12] M.R. Rani, K. Prakasan, R. Rudramoorthy, "Study of different joints for ultrasonic welding of semicrystalline polymers," Experimental Techniques, July/August, pp. 36-42, 2009.

[13] J.H. Qiu, G.H. Zhang, Y.B. Wu, "Proposal of ultrasonic welding technique and weld performances applied to polymers," Polymer Engineering and Science, September 2009, pp. 1755-1759.

[14] C.P. Buckley, J.J. Wu, D.W. Haughie, "The integrity of welded interfaces in ultra high molecular weight polyethylene: Part 1-Model,” Biomaterials, June 2006, pp. 3178-3186.

[15] D.W. Haughie, C.P. Buckley, J.J. Wu, "The integrity of welded interfaces in ultra high molecular weight polyethylene: Part 2-Interface toughness,” Biomaterials, July 2006, pp. 3875-3881.

ISSN: 0010-8189

(C) CONVERTER 2020 
[16] Y.B. Sun, Y. Luo, X.D. Wang, "Pressure-adaptive ultrasonic precision coupling of polymer MEMS devices," Optics and Precision Engineering, vol. 5, pp. 1189-1195, 2010.

[17] Y. Luo, M.M. Zhang, Y.B. Sun, X.D. Wang, Micro energy director array for ultrasonic precise sealing of polymer MEMS device. Optics and Precision Engineering, vol. 9, pp. 754-761, 2011.

[18] L. Tao, Y. Luo, Y.G. Zhang, et al., "Fabrication of micro energy director on polymethyl methacrylate substrate for micro ultrasonic bonding," Optics and Precision Engineering, vol. 17, no. 6, pp. 1373-1378, 2009.

[19] A.K. Sen, A. Raj, U. Banerjee, S.R. Iqbal, "Soft Lithography, Molding, and Micromachining Techniques for Polymer Micro Devices," Methods Mol Biol, vol. 1906, pp. 13-54. doi: 10.1007/978-1-4939-8964-5_2. PMID: 30488383, 2019.

[20] Y.G. Zhang, "Research on Ultrasonic Fusion Bonding Technology for Polymer Microfluidic Chips," Dalian: Dalian University of Technology, 2009. 